\title{
DIFFERENTIAL GEOMETRY AND HYDRODYNAMICS OF SOLITON LATTICES
}

\author{
S.P. NOVIKOV
}

\section{INTRODUCTION}

I am going to discuss here some results in the soliton theory of a Moscow group during the last years. The group of people who worked here include B.A. Dubrovin I.M. Krichever, S.P. Tsarev (and the present author). More details may be found in the survey article [1]. Modern needs in the large new classes of hydrodynamic type systems appear in connection with very interesting asymptotic method - so called "nonlinear analog of WKB-method", method of the slow modulations of parameters or "Whitham method" in the theory of solitons (see for example the book [2], chapter 4). This method is based on the large family of exact solutions $\varphi_{0}(x, t ; u)$ periodic or quasiperiodic in $x$ and $t$, of the form which we call "soliton lattice":

$$
\varphi_{0}(x, t ; u)=F\left(\eta_{0}+U x+V t ; u^{1}, \ldots, u^{N}\right) .
$$

Here $F\left(\eta_{1}, \ldots, \eta_{m} ; u^{1}, \ldots, u^{N}\right)$ is the function, which is $2 \pi$-periodic in each variable $\eta_{j}$ and depends on the $N$ parameters $u^{p}$. All quantities $\left(a_{j}, b_{k}, u^{p}\right)$ are constants and $\varphi_{0}(x, t ; u)$ satisfies some nonlinear P.D. equation

$$
\varphi_{t}(x, t)=K\left(\varphi, \varphi_{x}, \ldots \varphi^{(s)}\right),
$$

describing the propagation of solitons or some other nonlinear waves.

We have to have therefore some $N$-parametric family of invariant $m$-tori in the functional space of quasiperiodic functions. There are many such classical examples for $m=1$. For example in the case (0.2) is exactly the famous KDV-equation and for $m=1$ everybody knows the family of "knoidal waves" in elliptic functions

$$
\varphi_{0}\left(x, t ; u^{1}, u^{2}, u^{3}\right)=-2 \partial_{x}^{2} \log \Theta\left(k x+\omega t+\eta_{0}\right)+c .
$$

Here we have $u^{1}=c, u^{2}=k, u^{3}=\omega$. We write KDV in the form (0.3)

$$
\varphi_{t}-6 \varphi \varphi_{x}+\varphi_{x x x}=0 .
$$

The algebro-geometric solutions (or finite-gap solutions) of KDV discovered in [3] and investigated by many authors present us the analogous families for any $m>1$, $N=2 m+1$ (see details in $[2,4]$ ) The analogs of these solutions are known for all systems, integrable by the famous "Inverse scattering transform"(IST): SG (SineGordon), NS (Nonlinear Schrödinger), KP (Kadomtsev-Petviashvili) and others. Consider now the function

$$
\varphi_{0}\left(\frac{S(X, T)}{\epsilon} ; u(X, T)\right) .
$$

Here $X=\epsilon x, T=\epsilon t, S_{X}=U, S_{T}=V$. In 1965 Whitham observed (in some cases $-m=1$, the system $(0.2)$ is a nondegenerate Lagrangian system or it is 
exactly the KDV) - that the function (0.4) satisfies equation (0.2J) plus something small $(\epsilon \rightarrow 0)$ if the "slow functions" $u^{p}(X, T)$ satisfy some "hydrodynamic type" equation:

$$
u_{T}^{p}=v^{p}{ }_{q}(u) u^{q}{ }_{X} .
$$

A lot of authors developed this idea since 1965 (see the papers [5]-[10]; more complete list of references may be found in [1]). Serious investigations for $m>1$ started after the discovery of "finite-gap solutions" (1974-1975) — see the papers [11]-[13].

The activity of our group in this area started in 1982-1983. Its investigations were mainly concentrated on the next problems:

Problem 1: Suppose the original system (0.2) is hamiltonian corresponding to some local field-theoretical hamiltonian formalism. Is the associated hydrodynamic type system (or the "averaged system") (0.5) also hamiltonian? How to construct explicitly its coefficients using the hamiltonian formalism of the original system $(0.2)$ ?

Problem 2: Which kind of boundary problems for the equations of hydrodynamic type (0.5) appear from the "Nonlinear WKB" method? Is it possible to investigate them directly inside of the theory of the $1^{\text {st }}$ order systems as a new kind of hydrodynamics?

Problem 3: Suppose the original system (0.2) is completely integrable by the ISTmethod (Inv. Scatt. Transform). Is the averaged system (0.5) completely integrable? How to construct its solutions explicitly?

Problem 1 was solved by the present author and B.A. Dubrovin ([14]). This solution leads to the discovery of some deep and natural differentially-geometric structure for the hamiltonian hydrodynamic type equations (0.5), which does not exist for the generic hydrodynamic type equations (0.5) and was not observed during the 100 years of their investigations starting from Riemann. After that the Problem 3 (on the level of local differential geometry) was solved by S.P. Tsarev in his thesis (see $[15,16])$. The effective global algebro-geometric construction of some important solutions was found later by Krichever [17].

Problem 2 was investigated numerically by Avilov, Krichever and the present author ([18, 19]), for $m=1$ only. Very interesting classes of "multivalued functions" appear in the hydrodynamics of soliton lattices, but nothing rigorous was proven here.

In Appendix we discuss some beautiful restrictions on the initial data for the KDV-hierarchy which lead to rapidly oscillating functions and therefore to the "Nonlinear WKB". They have appeared recently in the so-called matrix models in the form of the "string equation".

We shall use the notation H.T. and P.B.H.T. for hydrodynamic type and Poisson brackets of hydrodynamic type respectively. Also P.B. denotes Poisson brackets, SH denotes semi-Hamiltonian, AFG denotes averaged finite gap solutions.

\section{Differential Geometry and Hydrodynamic Type Poisson Brackets}

1.1. Basic definitions. It will be most convenient to explain these ideas starting from the general geometric definitions. Let $X=\left(x_{1}, \ldots, x_{n}\right), n=$ "dimension of the space" and $x_{1}=x$ for $n=1$. Let $M$ be some $N$-dimensional manifold, where $N$ is the "number of components" and $u=\left(u^{1}, \ldots, u^{N}\right)$ are some local coordinates on $M$. The "hydrodynamic structure" on $M$ is by definition some collection of 
tensor fields numerated by the same indices as the $X$-space coordinates $v_{q}^{p, \alpha}(u)$. Definitions D1-D4 give us the base of geometric theory.

D1: The H.T. system is defined by the tensor fields $v_{q}^{p, \alpha}$ in the form

$$
u_{t}^{p}=v_{q}^{p, \alpha}(u) u_{\alpha}^{q},
$$

for the fields $u(x)$ belonging to some functional space of mappings $X \rightarrow M$.

D2: The Riemann invariants for the system (1.1) are such coordinates $u^{1}, \ldots u^{N}$ (if they exist) that all matrix fields $v_{q}^{p, \alpha}(u)$ are diagonal

$$
v_{q}^{p, \alpha}=v^{p, \alpha} \delta_{q}^{p}
$$

For $(N=2, n=1)$ the Riemann invariants always exist for hyperbolic systems (i.e. all individual matrices $v_{q}^{p, \alpha}\left(u_{0}\right)$ are equivalent to diagonal ones).

D3: The hydrodynamic quantities are such local functional on the space of fields $u(x)$, whose densities do not depend of the derivatives:

$$
J=\int j(u(x)) d x .
$$

The most important new definition of the paper [14] is the next one.

D4: The local homogeneous hydrodynamic type Poisson bracket is defined by the formula:

$$
\left\{u^{p}(x), u^{q}(y)\right\}=g^{p q, \alpha}(u(x)) \partial_{\alpha} \delta(x-y)+b_{r}^{p q, \alpha}(u(x)) \partial_{\alpha} u^{r}(x) \delta(x-y)
$$

for some functions $g^{p q, \alpha}(u), b_{r}^{p q, \alpha}(u)$ in the local coordinates $(u)$.

The H.T. hamiltonian (1.3) generates the H.T. system (1.1) using the Poisson bracket (1.4). If $H=\int h(x) d x$ we have

$$
\begin{aligned}
& u_{t}^{p}=g^{p k} u_{x}^{l} h_{; k ; l}, \\
& v_{q}^{p}=g^{p k} \nabla_{k}\left(\partial_{q} h\right) .
\end{aligned}
$$

The P.B. operation (1.3) has to be bilinear and skew symmetric; it has to satisfy Leibnitz and Jacobi identities. As a consequence of these requirements our quantities $g^{p q, \alpha}(u)$ should transform as symmetric tensors on $M$ for each $\alpha$ and $b_{k}^{p q, \alpha}$ should transform as $g^{p r, \alpha} \Gamma_{r k}^{q, \alpha}(u)$ for some Christoffell symbols $\Gamma$ on $M$ under the local changes of coordinates $u(w)$. The connections $\Gamma_{r k}^{q, \alpha}$ are compatible with the "metric" $g^{p q, \alpha}$ (for $\operatorname{det}\left(g^{p q, \alpha}\right) \neq 0$ ), if torsion and curvature are equal to zero.

There exist therefore some new coordinates $(w)$ such that

$$
\begin{aligned}
g^{p q, \alpha} & =\text { const }, \\
\Gamma_{r k}^{q, \alpha} & =0
\end{aligned}
$$

(for one value of $\alpha$ only!). For $n=1$ it gives us the complete invariant for the local classification of P.B.T.H. with $(\operatorname{det} g \neq 0)$-signature of the metric $g$.

So the canonical form of the nondegenerate P.B.H.T. is the generalized GardnerZakharov-Faddeev's $\delta^{\prime}$-bracket for $n=1$.

For $n>1$ we may kill Christoffell symbols for one value of $\alpha=1$ only; after that (as the present author, Dubrovin and Mokhov proved in $[20,21]$ ) all other "metrics" will be linear (may be nonhomogencous) functions in that variables $\left(u^{1}, \ldots, u^{N}\right)$, all other $b_{r}^{p q, \alpha}$ will be equal to const for $\alpha \neq 1$. Here we should have $\operatorname{det} g^{p q, 1} \neq 0$.

Such structures are invariant under the affine transformations of coordinates

$$
u=A w+u_{0} .
$$


1.2. Special P.B.H.T. Lie algebras. In the case when all metrics may be linear in $u$ we have

$$
\begin{array}{r}
g^{p q, \alpha}=g_{0}^{p q, \alpha}+C_{k}^{p q, \alpha} u^{k}, \\
b_{k}^{p q, \alpha}=\mathrm{const}, \quad g_{0}^{p q, \alpha}=\mathrm{const}, \\
C_{k}^{p q, \alpha}=b_{k}^{p q, \alpha}+b_{k}^{q p, \alpha} .
\end{array}
$$

The linear (homogeneous) part of such P.B.H.T. determines some very interesting class of infinite-dimensional Lie algebras ("hydrodynamic algebras"): for two vector-functions $f(x)$ and $g(x)$ with $N$ components $f_{p}, g_{q}$ we may define the commutator in the "local translation-invariant first-order Lie algebra" or hydrodynamic algebra

$$
[f, g]_{r}=\left[\left(\partial_{\alpha} f_{p}\right) g_{q}-\left(\partial_{a} g_{p}\right) f_{q}\right] b_{r}^{p q, \alpha} .
$$

For $n=1$ these algebras were investigated in [22]. The hamiltonian formalism of classical liquid (including the magnetohydrodynamics and superfluid systems ad the references to surveys of Khalatnikov, Dzyaloshinsky and other physicists especially from Landau Institute) in connection with some special Lie algebras was discussed in [23].

It is useful to introduce new algebra $B$ as a multiplication in the $N$-space $M$ with basis $e^{1}, \ldots, e^{N}$

$$
e^{p} \circ e^{q}=b_{k}^{p q} e^{k} .
$$

For the functions $f(x)=f_{p}(x) e^{p}$ and $g(x)=g_{q} e^{q}$ we write (1.8) in the form

$$
f^{\prime} \circ g-g^{\prime} \circ f
$$

using the multiplication $\circ$ in algebra $B$ - see (1.9). In that case $(n=1)$ our formula (1.8) determines correctly the Lie algebra $L_{B}$ if and only if for any three elements of the algebra $B$ the next identities are true: if $L_{a}(b)=a \circ b, R_{b}(a)=a \circ b$ we should have

$$
\begin{aligned}
{\left[L_{a}, L_{b}\right] } & =0, \\
{\left[R_{b}, R_{c}\right] } & =R_{b \circ c-c \circ b} .
\end{aligned}
$$

The constant part of P.B. (1.7) $g_{0}^{p q, \alpha}$ determines some 2-cocycle on the Lie algebra $L_{B}$ for $n=1$

$$
\gamma[f, g]=\int f_{p}^{\prime} g_{0}^{p q} g_{q}(x) d x .
$$

In general, local translational-invariant 2-cocycles of the type $\tau=0,1,2,3$, on the algebra $L_{B}$ may be defined by the formula

$$
\gamma[f, g]=-\gamma[g, f]=\int \gamma_{0}^{p q} f_{p}^{(\tau)}(x) g_{q}(x) d x .
$$

We have $\tau=1$ in the case (1.10). We may have nontrivial cocycles for $(\tau=$ $0,1,2,3)$ only. The classical Gelfand-Fuchs cocycle for $B=R, \tau=3$ generates the well-known Virasoro algebra - extension of the algebra of vector fields on the circle $L_{R}$ ). Formula (1.11) determines some cocycles of the type $\tau=3$ for commutative algebra $B$ if the symmetric form $\gamma_{0}^{p q}$ determines scalar product $\langle$,$\rangle on B$ with the next properties

$$
\langle a b, c\rangle=\langle a, c b\rangle
$$


If $1 \in B$ and the form is nondegenerate we come to the classical Frobenius algebras. In that case we always have

$$
\gamma_{0}^{p q}=C_{k}^{p q} u_{0}^{k}
$$

for some point $u^{0}$. The case $\tau=1$ is also very important. Symmetric form in that case is such that (1.12) is also true but the algebra $B$ may be noncommutative.

The corresponding cocycle of the type $(\tau=1)$ is cohomologous to zero if (1.13) is true. Interesting examples presents us P.B.H.T. for the classical gas dynamics (for $n=1)$. We have here $N=3$ - the densities of momenta, of mass and of entropy are our basic fields. Let $f(x)=p(x) e^{1}+\rho(x) e^{2}+s(x) e^{3}, e^{1}=e, e^{2}=a, e^{3}=b$. For the multiplication in algebra $B$ we have (we omit the symbol o)

$$
e^{2}=e, \quad e a=a, \quad e b=b, \quad b e=a e=a^{2}=b^{2}=a b=b a=0 .
$$

The "metric" $g^{p q}(u)$ here has the rank 2. This P.B.H.T. does not satisfy the nondegeneracy condition

$$
\operatorname{det} g^{p q}=0
$$

But there are nontrivial cocycles for $\tau=1 ; g_{0}^{p q}$ should be such that

$$
\langle e, B\rangle=0
$$

Therefore $g_{0}^{p q}$ is some arbitrary two-by-two symmetric matrix concentrated on the subspace $(2,3)$. The perturbed P.B.H.T. has nondegenerate metric

$$
g^{p q}(u)=g_{0}^{p q}+C_{r}^{p q} u^{r}
$$

which has zero curvature.

The case $\tau=2$ may also be interesting. The form here and the corresponding scalar product should be such that

$$
\begin{gathered}
\langle a, b\rangle=-\langle b, a\rangle, \\
\langle a c, b\rangle=-\langle a, b c\rangle,
\end{gathered}
$$

Any cocycle of the type $\tau$ generates the extension of P.B. where the extended P.B. is equal to original P.B.H.T. plus $\left(g_{0}^{p q} \delta^{(\tau)}(x-y)\right)$.

The theory of extensions for Lie algebras $L_{B}$ was constructed in $[22,23]$. As Zelmanov proved in [24] the algebra $B$ (over $C$ ) with $N>1$ contains some ideal $I \subset B$ such that $I^{2}=0$. Therefore all our algebras $B$ may be constructed from the factor-algebras $A$ of $B$ by $I$ and the $A$-modules $I$ using the cocycles of the work [22]. There was some nice example of the algebra $B$ in the paper [25]; some of their algebraic properties were discussed by S. Gelfand.

1.3. Liouville structures. Suppose $n=1$.

D.6: The coordinates $u^{1}, \ldots, u^{N}$ are Liouville for the P.B.H.T. (1.7) if there exist some tensor fields $\gamma^{p q, \alpha}(u)$ such that

$$
\begin{aligned}
g^{p q, \alpha} & =\gamma^{p q, \alpha}+\gamma^{q p, \alpha}, \\
b_{r}^{p q, \alpha} & =\frac{\partial \gamma^{p q, \alpha}}{\partial u^{r}} .
\end{aligned}
$$

Example: for the brackets (1.6) linear in $u$ the same coordinates $u$ are Liouville

$$
\gamma^{p q, \alpha}=b_{r}^{p q, \alpha} u^{r}+\text { const. }
$$

The Liouville structure is covariant under the affine transformations only. 
D7: We call the coordinates strongly Liouville for P.B.H.T. if its restriction to any group $\left(u^{i_{1}}, \ldots, u^{i_{k}}\right)$ of coordinates (after affine transformation) correctly determines some P.B.H.T. with Liouville structure. As the present author and Dubrovin observed in [1] they appear from the "Nonlinear WKB" for the hydrodynamic of soliton lattices. For $n=2$ any Liouville structure is strong, but for $N>2$ it is not so. The P.B. of the classical gas dynamic is strongly Liouville in the coordinates $(p, \rho, s)$. A nice example comes from a relativistic liquid. Its energy-momentum tensor $\left(\left(t_{i j}\right)=T ; i, j=0,1\right)$ is symmetric and

$$
t_{00}=\varepsilon, \quad t_{01}=p, \quad \operatorname{tr} T=t_{00}-t_{11}=q .
$$

The H.T. equations have the form

$$
\partial_{i} T_{j}^{i}=0
$$

The eigenvalues of $T$ in the Minkovsky metric are the density of inner energy $\varepsilon_{0}$ and pressure $P$. We have $q=\varepsilon_{0}-P$. With state equation $F\left(\varepsilon_{0}, P\right)=0$ we obtain the complete system. The P.B.H.T. for it is such that $H=\int t_{00} d x$ and

$$
\gamma^{i j}=\left(\begin{array}{ll}
t_{01} & t_{00} \\
t_{11} & t_{10}
\end{array}\right)
$$

There is discussion in [1] about some interesting generalizations of P.B.H.T.: higher order analogs, nonhomogeneous brackets, discrete analog (which leads to the linearized Yang-Baxter equations with very interesting additional structure).

\section{Weakly Deformed Soliton Lattices and Their Hamiltonian HYDRODYNAMICS}

Suppose the original system (0.2) is hamiltonian corresponding to some local field-theoretical P.B. $\{,\}_{0}$ and hamiltonian $H$

$$
\begin{aligned}
H & =\int h\left(\varphi, \varphi_{x}, \ldots\right) d x, \\
\{\varphi(x), \varphi(y)\}_{0} & =\sum_{k=0}^{L} B_{k}\left(\varphi(x), \ldots \varphi^{\left(n_{k}\right)}(x)\right), \\
\varphi_{t} & =\{\varphi(x), H\}_{0} .
\end{aligned}
$$

Consider any family of the "soliton lattices" (0.1) which is nondegenerate

$$
U=\left(k_{1}, \ldots, k_{m}\right), \quad \operatorname{rk}\left(\frac{\partial k_{i}}{\partial u^{j}}\right)=m .
$$

It means that the first $m$ parameters may be $k_{p}$ and $N \geqslant m$. Suppose also that there are at least $N$ independent local commuting integrals

$$
\begin{gathered}
I_{p}=\int P_{p}\left(\varphi, \varphi_{x}, \ldots, \varphi^{\left(l_{p}\right)}\right) d x, \\
\left\{I_{p}, I_{q}\right\}_{0}=0 .
\end{gathered}
$$

For the densities we have some finite sum

$$
\left\{P_{p}, P_{q}\right\}_{0}=\sum_{k} A_{k}^{p q}\left(\varphi(x), \ldots, \varphi^{\left(l_{k}\right)}(x)\right) \delta^{(k)}(x-y) .
$$


From the commutativity $(2.5)$ we deduce that $\int A_{0}^{p q}=0$ for any field $\varphi$. It mean that

$$
A_{0}^{p q}(\varphi, \ldots)=\partial_{x} Q^{p q}(\varphi, \ldots)
$$

Let us introduce the physical coordinates $u$, metric and Christoffel symbols by the formulas:

$$
\begin{aligned}
g^{p q}(u) & =\bar{A}_{1}^{p q}=(2 \pi)^{-m} \int_{T^{m}} A_{1}^{p q}\left(F, F^{\prime}, \ldots\right) d^{m} \eta \\
\gamma^{p q}(u) & =\bar{Q}^{p q}=(2 \pi)^{-m} \int_{T^{m}} Q^{p q}\left(F, F^{\prime}, \ldots\right) d^{m} \eta \\
b_{r}^{p q} & =\frac{\partial \gamma^{p q}}{\partial u^{r}}, \quad u^{q}=\bar{P}_{q} .
\end{aligned}
$$

Here we have

$$
F^{\prime}=k_{j} \frac{\partial F}{\partial \eta_{j}}, \ldots
$$

and $F$ is from $(0.1)$

Our theorem states that that the metric (2.9) has zero curvature and the corresponding P.B.H.T. in physical coordinates (2.8) has the strongly Liouville form (2.10). The averaged H.T. equation is the hamiltonian H.T. system in the bracket (2.8)-(2.10). Its hamiltonian is H.T. quantity whose density exactly coincides with the averaged density of energy

$$
\begin{aligned}
\varepsilon & =\bar{h}(u), \\
H & =\int \varepsilon d x .
\end{aligned}
$$

All averaged densities $\bar{P}_{q}=u^{q}$ determine the H.T. conservative quantities

$$
I_{p}=\int u^{p} d x
$$

The averaged P.B.H.T. of them are equal to zero. This procedure may be considered as an alternative definition of the averaged system. Our works were dedicated to the studying of that procedure. Some other people investigated this question - in which cases this equations lead to the nonlinear WKB-type approximation (see for example [11])? Our goal was to investigate the formal properties of the resulting H.T. systems (including their hamiltonian formalism, exact integrability and also the boundary conditions for them natural in the physical problems).

The special case of nondegenerate Lagrangian system was investigated by Whitham in 1965 for $m=1$. The formal properties for any $m>1$ are the same here. In that case we don't need the local integrals as above. For example it is important for some nonintegrable systems like the perturbed NS

$$
i \psi_{t}=\psi_{x x}+V\left(|\psi|^{2}\right) \psi
$$

Here $m=2, N=4$; but we have generically here only three conservative quantities. In the integrable case we have more, of course. 
The Lagrangian averaging leads to the H.T. systems in the form (2.12):

$$
\begin{aligned}
& v_{t}^{j}=\partial_{x}\left(\frac{\delta H}{\delta w^{j}(x)}\right), \\
& w_{t}^{j}=\partial_{x}\left(\frac{\delta H}{\delta v^{j}(x)}\right) .
\end{aligned}
$$

Here we have $j=1, \ldots, m, N=2 m,(u)=(v, w)$. In that case the metric has a constant form

$$
g^{p q}=\left(\begin{array}{ll}
0 & 1 \\
1 & 0
\end{array}\right)
$$

Its signature is $(m, m)$. In the variables $(v, y), y=\int w d x$ the system (2.12) will be Lagrangian. This form exactly corresponds to the classical "Clebsch variables" in hydrodynamics. A lot of people considered the hamiltonian formalism of H.T. systems in the form (2.12) only (see for example [26]).

It is natural to suppose that for the hamiltonian system (0.1) the subspace (0.1) presents some finite-dimensional system, integrable in the strict sense of Liouville or some family of such systems depending from $N-2 m$ parameters. Suppose $J_{1}, \ldots, J_{m}$ are the action variables. In [14] we formulated a theorem that the quantities

$$
u^{j}=k_{j}, \quad u^{m+j}=J_{j}, \quad j=1, \ldots, m,
$$

have the averaged P.B. of the form (2.13). This observation for $m=1$ was made in the work of Hayes [9]. Up to now this theorem was proven for the integrable systems only.

Motivated by the last applications of the action variables the present author, Veselov and Dubrovin investigated them and constructed a theory of "algebrogeometric" finite dimensional P.B. for the systems, integrable by the method of Riemann surfaces (see [27, 28]; these ideas were initiated in some calculations of the papers $[29,30])$.

\section{Integrability of the Hamiltomian H.T. Systems}

3.1. Riemann Invariants for the Hamiltonian H.T. systems. Integrability and Differential Geometry. The Riemann invariants are known for some classical systems of gas dynamics more than 100 years. Their existence for $N>2$ is the true sign for some degeneracy in the system. For $(N=2, n=1)$ they always exist. The transformation to the inverse functions $x\left(u^{1}, u^{2}\right), t\left(u^{1}, u^{2}\right)$ linearizes the H.T. system. This is well-known "hodograph transformation". Is there any generalization of the "hodograph" for $N>2$ ? This problem was solved by Tsarev in his thesis [12] in the process of investigating the diagonal hamiltonian H.T. systems. The conjecture about the integrability of that class was formulated by the present author and posed to Tsarev as a problem.

Tsarev's main theorem states that such systems are integrable in the strict sense of Liouville at least in the class of monotonic functions. He found also a natural analog of the "hodograph" method for the construction of exact solutions.

Let us consider the diagonal hamiltonian H.T. system

$$
u_{t}^{p}=v^{p}(u) u_{x}^{p}, \quad v^{p} \neq v^{q}
$$


corresponding to some hamiltonian

$$
H=\int h(u) d x
$$

and P.B.H.T. (1.4) for $n=1$ with nondegenerate metric $g^{p q}(u)$. It is easy to prove that the metric will be also diagonal

$$
g^{p q}=g^{p}(u) \delta_{q}^{p}
$$

in the same coordinates (but not constant).

The classification of the orthogonal coordinates in the euclidean space is the classical problem. E. Cartan proved that they depend on the $N(N-1)$ independent functions of 2 variables.

As Tsarev observed, all diagonal H.T. systems which are hamiltonian in the same P.B.H.T. commute with each other. We have:

$$
\begin{aligned}
u_{t}^{p} & =w^{p}(u) u_{x}^{p}, \quad H_{2}=\int h_{2}(u) d x, \\
\Gamma_{k i}^{k} & =\left(\frac{\partial_{i} w^{k}}{w^{i}-w^{k}}\right)=\left(\frac{\partial_{i} v^{k}}{v^{i}-v^{k}}\right) m \\
2 \Gamma_{k i}^{k} & =\partial_{i} \log \left|g_{k}\right| .
\end{aligned}
$$

All collections of the systems (3.3) depend on $N$ functions of 1 variable. They may be found "in principle" from the equation (3.3) on the level of local differential geometry. The next consequence will be important:

$$
\partial_{i}\left(\frac{\partial_{j} w^{k}}{w^{j}-w^{k}}\right)=\partial_{j}\left(\frac{\partial_{i} w^{k}}{w^{i}-w^{k}}\right) .
$$

Definition. We shall call the diagonal H.T. system a semihamiltonian (SH) one if (3.4) is valid for its coefficients.

The class of SH systems is larger than the class of the diagonal hamiltonian ones. For $(N=2)$ all H.T. systems are diagonalizable but the hamiltonian subclass depends on 3 functions only. SH systems have the most important property of the hamiltonian H.T. systems: They are deeply connected with some diagonal metric, determined from the equation (3.3), but may have nonzero curvature if the $\mathrm{SH}$ system is really nonhamiltonian).

The integration process works for all SH systems: We have a large collection of commuting SH systems from (3.3). Each system of that class generates some exact solution. Consider the functions $u^{p}(x, t)$ obtained from the equation (3.5):

$$
v^{p}(u(x, t)) t+x=w^{p}(u) .
$$

They satisfy to the original equation (3.1). It gives locally the complete solution of (3.1).

There is also a construction of a large family of conserved quantities for the $\mathrm{SH}$ systems. It was known for $N=2$ many years. For the hamiltonian systems the commuting flows and the conserved quantities are in a natural one-to-one correspondence. It is unclear now - is there any hamiltonian explanation for the $\mathrm{SH}$ class? 
3.2. Soliton lattices for the integrable systems. The applications of these methods to any concrete integrable system is nontrivial problem. Consider for example KDV in the form (0.3). Even the simplest case $m=1, N=3$ leads to the highly nontrivial "Whitham system" after averaging. Whitham observed that it admits the Riemann invariants $\left(r_{j}\right)$. Its analytical form is:

$$
\left(r_{j}\right)_{T}=v^{j}(r)\left(r_{j}\right)_{X}
$$

where

$$
\begin{gathered}
-3 v^{j}=r_{1}+r_{2}+r_{3}-2 f_{j}, \\
f_{1}=\left(r_{2}-r_{1}\right)\left(\frac{K}{K-E}\right), \quad f_{2}=\left(r_{3}-r_{1}\right)\left(\frac{\left(1-s^{2}\right) K}{E-\left(1-s^{2}\right) K}\right), \\
f_{3}=\left(r_{1}-r_{3}\right) \frac{\left(1-s^{2}\right) K}{E}
\end{gathered}
$$

and $K(s), E(s)$ are the standard "complete elliptic integrals",

$$
s^{2}=\frac{r_{2}-r_{1}}{r_{3}-r_{1}}, \quad v_{1} \leqslant v_{2} \leqslant v_{3}, r_{1} \leqslant r_{2} \leqslant r_{3} .
$$

On the boundary our family degenerates to a constant solution of KDV for $r_{2}=r 1$ and to soliton for $r_{3}=r_{2}$. The result about the existence of the Riemann invariants was generalized in 1980 ([12]) to the families of finite-gap solutions $m \geqslant 1$, $N=2 m+1$ : they are exactly the branching points of Riemann surfaces $\Gamma$ and the endpoints of the spectrum of corresponding Schrodinger operator with periodic (quasiperiodic) potential on the line:

$$
\left(r_{j}\right)_{T}=v^{j}(r)\left(r_{j}\right)_{X}, \quad j=1, \ldots, 2 m+1 .
$$

The same results are valid for the systems SG and NS. The family of finite -gap solutions has a form for KDV:

$$
\varphi(x, t ; u)=-2 \partial_{x}^{2} \log \Theta\left(U x+V t+\eta_{0} \mid B\right)+C .
$$

Here we have the Riemann surface

$$
\begin{gathered}
\Gamma: \mu^{2}=\prod\left(\lambda-r_{j}\right), \quad\left(r_{j} \text { real }\right), \\
U=\left(k_{1}, \ldots, k_{m}\right), \quad V=\left(\omega_{1}, \ldots, \omega_{m}\right), \quad B=\left(b_{i j}\right), \\
\oint_{b_{i}} \Omega_{j}=b_{i j}, \quad \oint_{a_{j}} \Omega_{k}=2 \pi i \delta_{j k}, \quad \oint_{b_{j}} d p(\lambda)=k_{j}, \quad \oint_{b_{j}} d q(\lambda)=\omega_{j} .
\end{gathered}
$$

Here $\omega_{j}$ are the $1^{\text {st }}$ kind differentials and $d p, d q$ are the second kind ones on the Riemann surface $\Gamma$ normalized as in (3.11):

$$
\begin{gathered}
\oint_{a_{j}} d p=\oint_{a_{j}} d q=0, \\
d p=d k+\text { regular, } \quad d q=d\left(k^{2}\right)+\text { regular, } \\
z=k^{-1}=\lambda^{-\frac{1}{2}} \rightarrow 0, \quad a_{j}=\left(\left[r_{2 j-1}, r_{2 j}\right] ; \pm\right) .
\end{gathered}
$$

The multivalued functions $p(\lambda), q(\lambda)$ are the "quasi-momentum" and "quasienergy", $a_{k}, b_{k}$ - canonical basis of cycles:

$$
a_{k} \circ a_{j}=b_{k} \circ b_{j}=0, \quad a_{k} \circ b_{j}=\delta_{k j} .
$$


It is well-known that the Kruskal integrals exactly coincide with the asymptotic coefficients

$$
\begin{aligned}
p(\lambda) & =\sqrt{\lambda}+\sum_{s=0}^{\infty} I_{s} \frac{1}{(2 \sqrt{\lambda})^{2 s+1}}, \\
I_{s} & =\int P_{s}(\varphi, \ldots) d x .
\end{aligned}
$$

Here $P_{0}=\varphi, P_{1}=\frac{\varphi^{2}}{2}, P_{2}=\frac{\varphi_{x}^{2}}{2}-\varphi^{3}, \ldots$

As it was observed in [12], the averaged H.T. system has the nice algebraic form

$$
d_{T} p(\lambda)=d_{X} q(\lambda)
$$

The generalization of the form (3.14) to KP was found by Krichever [17] who was able also to develop a "Nonlinear WKB"-method for KP (this is more difficult because KP is a non-local evolution system). The averaged densities $\bar{P}_{s}$ generate the conserved quantities

$$
I_{s}=\int u^{s} d x, \quad s=1,2, \ldots
$$

Any linear combination of them is also a conserved quantity

$$
I=\sum_{j} c_{j} I_{j}
$$

So we have a huge family of conserved H.T. quantities after averaging

$$
\sum_{p} u^{p}\left(r_{1}, \ldots, r_{2 m+1}\right)
$$

Any independent group of $(2 m+1)$ of such quantities determines the "physical coordinates"

$$
w^{p}=\sum_{j \geqslant 0} c_{j}^{p} u^{j}\left(r_{1}, \cdots r_{2 m+1}\right),
$$

such that the H.T.P.B. has a strongly Liouville form. This property gives us some characterization of the averaged P.B. for the integrable KDV-type systems.

All quantities $v^{p}$ determine some exact solutions by the Tsarev's procedure (above). We call these solutions "the averaged finite-gap solutions" (AFG).

For the basic quantities $u^{q}=\bar{P}_{q}$ the AFG-solutions are self-similar as it was observed by Krichever. There is a nice formula:

$$
v^{j}(r)=\left(\frac{d p}{d q}\right)_{\lambda=r_{j}} .
$$

The AFG-solution generated by the averaged Kruskal integral $\int u^{q} d x$ using the hamiltonian formalism and (3.5) may be written in the form:

$$
\begin{aligned}
r_{p}(X, T) & =T^{\gamma} f_{p}\left(X T^{-1-\gamma}\right), \\
\gamma & =\frac{1}{q-2}, \quad q=3,4, \ldots
\end{aligned}
$$


(details see in [1]). For $q=4, m=1$ computations were done in [31]. We obtain from $(3.5)$

$$
\begin{gathered}
w^{p}=\frac{1}{35}\left[\left(3 v^{p}-a\right) f_{p}+f\right], \\
f=5 a^{3}-12 a b+c, \\
a=\sum_{p=1}^{3} r_{p}, \quad b=\sum_{p<q} r_{p} r_{q}, \quad c=r_{1} r_{2} r_{3}, \\
f_{p}=\frac{\partial f}{\partial r^{p}} .
\end{gathered}
$$

The system $(3.18)$ is nondegenerate in the domain $\Delta=\left(z_{-}, z_{+}\right)$:

$$
z_{-}=-\sqrt{2}, \quad z_{+}=\frac{+\sqrt{10}}{27}, \quad z=x t^{-\frac{3}{2}} .
$$

Consider the curve $r(x, t)=t^{\frac{1}{2}} l(z)$ determined outside of $\Delta$ by the equation

$$
x+6 r t=(r)^{3} \text {. }
$$

It will be by definition the $C^{0}$-continuation of the 3 -valued curve $\left(r_{1}, r_{2}, r_{3}\right)$ at any moment $t$ equal to constant.

We have the "boundary condition":

$$
\begin{array}{ll}
r_{2}\left(x_{-}\right)=r_{1}\left(x_{-}\right), & r_{3}\left(x_{-}\right)=r\left(x_{-}\right), \\
r_{3}\left(x_{+}\right)=r_{2}\left(x_{+}\right), & r_{1}\left(x_{-}\right)=r\left(x_{+}\right) .
\end{array}
$$

So the complete $C^{0}$-curve $r(z)$ is such that

$$
r(z)=\left(r_{1}, r_{2}, r_{3}\right), \quad z \in \Delta .
$$

The "dispersive shock wave" $r(x, t)=t^{\frac{1}{2}} l(z)$ which was found numerically by Gurevitch and Pitaevsky in 1973 and which we call "GP"-solution belongs to $C^{1}$ class in the points $\left(z_{-}, z_{+}\right)$and may have singularity in some point $z_{0}, l_{2}\left(z_{0}\right)=0$. After proving that in fact the functions (3.18)-(3.20) are $C^{1}$ we shall come to the result that our formulas present the "dispersive shock wave" exactly (and there is no singularity in the point $r_{2}=0$ ). This important difference in the definition of the functional classes has been missed in [31] as the present author pointed out to Krichever and Potemin.

Consider the meromorphic form $d s(\lambda)$ on the surface $\Gamma$ such that:

$$
\oint_{a_{j}} d s=0, \quad d s=\sum_{p \geqslant 1} c_{p} d\left(k^{2 p-1}\right)+(\text { regular }) .
$$

All collection of the "averaged finite-gap" solutions may be obtained from the next equation of Krichever:

$$
\begin{gathered}
(X d p+T d q-d s)_{\lambda=r_{p}}=0, \\
p=1, \ldots, 2 m+1 .
\end{gathered}
$$

Here we have $c_{4}=0, c_{p}=0(p \neq 4)$ in the GP-case above. More general class of the forms $d s$ with some jumps along the curves on the surface $\Gamma$ leads to the general solution of the averaged H.T. system of hydrodynamics of soliton lattices for KDV. 
3.3. Time evolution of the multivalued functions. Numerical investigations. Hydrodynamics of soliton lattices based on the Whitham type equations cannot be realized on the ordinary spaces of one-valued functions in the physically interesting cases. We should define the time evolution for the multivalued functions. (The same conclusion may be deduced from the results of Lax, Levermore and Venakides $[32,33]$.) We constructed in the works $[18,19]$ the class of special 1 - and 3-valued functions for the investigation of stability of the "dispersive shock wave" which should be realized also as the asymptotics $t \rightarrow \infty$.

Consider now 2 classes of multivalued functions $r(x)$, one-valued for $|x| \rightarrow \infty$ :

Class 1: $r(x) \rightarrow A_{ \pm},|x| \rightarrow \infty$,

Class 2: $r(x) \sim x^{\frac{1}{3}},|x| \rightarrow \infty$.

We suppose that there is one and only one finite interval $\Delta$ for each function $r(x)$ such that it is one-valued outside $\Delta$ and 3 -valued inside $\Delta=\left[x_{-}, x_{+}\right]$. The branches $r_{p}$ of $r$ should be such that

$$
\begin{gathered}
r_{1}<r_{2}<r_{3} \quad(\text { inside } \Delta), \\
r_{1}\left(x_{-}\right)=r_{2}\left(x_{-}\right)=r_{-}<r_{3}, \quad r_{2}\left(x_{+}\right)=r_{3}\left(x_{+}\right)=r_{+}>r_{1} .
\end{gathered}
$$

The graph of the curve $r(x)$ should be $C^{1}$-smooth. The most important requirements are the asymptotics near the boundary of $\Delta$ :

$$
\begin{aligned}
& 0>x-x_{-}=\left[a_{+}+b_{+}\left(r-r_{+}\right)\right] f\left(1-s^{2}\right)+O\left(r-r_{+}\right)^{3}, \\
& 0<x-x_{-}=\left[a_{-}+b_{-}\left(r-r_{-}\right)\right]\left(r-r_{-}\right)^{2}+O\left(r-r_{-}\right)^{3} .
\end{aligned}
$$

The parameter $s^{2}$ was defined in (3.7) and

$$
f(u)=u \log \left(\frac{16}{u}+\frac{1}{2}\right), \quad u=1-s^{2} .
$$

Time evolution was realized numerically [18] for the functional classes above. Our conclusion is that for the $C^{1}$-small perturbations of the GP-solutions (in the both classes 1 and 2) the time dynamics is correctly defined for all $t>t_{0}$ and has self-similar GP-asymptotics for $t \rightarrow \infty$. For the class 1 this result may be rigorously deduced from the IST-method for KDV (see [34]). For the important class 2 connected with the "dispersive shock wave" it is probably impossible now.

The influence of small viscosity in the same classes was investigated in [19]. More details and discussion may be found in the survey [1].

\section{APPENDIX}

The situation of dispersive shock wave appeared recently in the so-called "quantum 2D-gravity", as you may find in the preprints of Douglas, Seiberg and Shenker, Molinari and Parisi (1990). After the results of Gross and Migdal, Brezin and Kazakov, Douglas and Shenker (the end of 1989) we know that the so-called renormalization group for the "matrix models" in some special continuum limit (for the large order of matrices) may be described as a KDV-type hierarchy:

$$
\frac{\partial L}{\partial t_{j}}=\left[A_{j}, L\right], \quad j \leqslant l-1, t_{0}=x .
$$

The critical points, corresponding to concrete conformal theories coupled with gravity should satisfy to the next equation (string equation)

$$
\left[A_{l}, L\right]=1
$$


(and $L$ should have very special asymptotic for $|x| \rightarrow \infty$; if $L=-\partial^{2}+u$ we have here exactly the KDV hierarchy and $u \sim x^{\frac{1}{l+1}}$.

For $l=1$ the equation (A.2) coincides with the classical "Painleve-1" equation it never was written before in the form (A.2)). For $l=2$ its asymptotic for the large $x$ is exactly like in the case of the "dispersive shock wave" (see the last part 3.3 of the paragraph 3 ). The asymptotic behavior of solution for $t \rightarrow \infty$ is written in $(3.18)-(3.20)$.

The present author and Krichever developed recently useful asymptotic methods (nonlinear WKB and linear WKB, using Riemann surfaces and Lax-type pairs) for the studying the equation (A.2), written in the form:

$$
[A, L]=\varepsilon 1 \text {. }
$$

$($ see $[36,37])$.

Very recently the present author investigated the equation (A.3) and found very interesting formula for the physically important "string solution". In the simplest nontrivial case (order of $A$ equal 3, order of $L$ equal 2, where the equation (A.3) is exactly the Painleve-1) the potential $u(x)$ has the form:

$$
\begin{aligned}
u & =2 \wp\left(\frac{\omega}{2} \| g_{2}, g_{2}\right), \\
2 g_{3}^{\prime} & =\varepsilon u, \\
g_{2} & =-\varepsilon x, \quad x \rightarrow-\infty .
\end{aligned}
$$

The quantity $2 \omega$ here is the real period. The basic periods are complex-adjoint. This equation may be obviously integrated by 1 quadrature. Our main conjecture in [36] was not true; the $\psi$-function is in fact multivalued on the Riemann surface. After the investigation I found that the conjecture has to be replaced by the formula (A.4). The analogous result is true for the operators of the higher order also. This work is still not finished: I still do not know-is this formula exact or only asymptotic for $x \rightarrow \infty$ ?

\section{REFERENCES}

[1] Dubrovin B.A., Novikov S.P., UMN (Russian Math. Surveys) 44 (6) 29-98 (1989).

[2] Novikov S.P., Manakov S.V., Pitaevsky L.P., Zakharov V.E., Theory of solitons. Plenum Press, New York (1984).

[3] Novikov S.P., Funk. Anal. Appl. 8 (3) 54-66 (1974).

[4] Dubrovin B.A., Matveev V.B., Novikov S.P., UMN, 31 (1) 55-136 (1976).

[5] Whitham C.B., a) J. Fluid. Mech., 22 (2) 273-283 (1965). b) Proc. Royal Society London, A139 283-291 (1965).

[6] Luke J.C. Proc. Royal Soc. London, A292 (1430) 410-412 (1966).

[7] Maslov V.P., Theor. Math. Phys., 1 (3) 378-383 (1969).

[8] Ablowitz M.J., Benney D.J., Studies Appl. Math. 49 (3) 225-238 (1970).

[9] Hayes W.D., Proc. Royal Soc. London, 332 199-221 (1973).

[10] Gurevitch A.V., Pitaevsky L.P., a) JETP 65 (2) 590-604 (1973). b) Letters of JETP, 17 (5) 268-271 (1973).

[11] Dobrokhotov S.Yu., Maslov V.P., Soviet Sci. Rev. (ser. C, Math. Phys. Rev.) 3 1-150 (1982).

[12] Flaschka H., Forest M.G., Mclaughlin D.W., Comm. Pure Appl. Math. 33 (6) 739-784 (1980).

[13] Flaschka H., Mclaughlin D.W., Studies Appl. Math., 68 (1) 11-59 (1983).

[14] Dubrovin B.A., Novikov S.P., DAN SSSR (= Soviet Math. Dokl.) 270 (4) $781-785$ (1983).

[15] Tsarev S.P., DAN SSSR, 282 (3) 534-537 (1985).

[16] Novikov S.P., UMN, 40 (4) 79-89 (1985).

[17] Krichever I.M., Funk. Anal. Appl., 44 (2) 37-52 (1989).

[18] Avilov V.V., Novikov S.P., DAN SSSR, 294 (2) 325-329 (1987). 
[19] Avilov V.V., Krichever I.M., Novikov S.P., DAN SSSR, 295 (2) 345-349 (1987).

[20] Dubrovin B.A., Novikov S.P., DAN SSSR, 279 (2) 294-297 (1984).

[21] Mokhov O.I., Funk. Anal. Appl., 22 (4) 92-93 (1988).

[22] Balinsky A.A., Novikov S.P., DAN SSSR 283 (5) 1036-1039 (1985).

[23] Novikov S.P., UMN, 37 (5) 3-49 (1982).

[24] Zelmanov E.I., DAN SSSR, 292 (6) (1987).

[25] Gelfand I.M., Dorfman I.A., Funk. Anal Appl., 13 (4) 13-30 (1979)

[26] Ercolani N., Forest M.G., Mclaughlin D.W., Montgomery R., Duke Math. J., 55 (4) $949-983$ (1987).

[27] Veselov A.P., Novikov S.P., a) DAN SSSR 266 (3) (1982). b) Trans. of Steklov Math. Inst. 165 49-61 (1984).

[28] Dubrovin B.A., Novikov S.P., DAN SSSR, 267 (6) 1295-1300 (1982).

[29] Flaschka H., Mclaughlin D.W., Progr. Theor. Phys., 55 (6) 438-456 (1976).

[30] Alber S.I., Preprint of the Institut of the Chemical Physics, Chernogolovka, AN SSSR, (1976).

[31] Potemin G.V., UMN, 43 (5) 211-212 (1988).

[32] Lax P.D., Levermore C.D., Comm. Pure Appl. Math., 36 253-290, 571-593, 809-830 (1983).

[33] Venakides S., a) AMS Transactions, 301 189-226. b) Comm. Pure Appl. Math., 38 125-155 (1985).

[34] Bikbaev R.V., Novokshenov V.Yu., Proc. of the third International Workshop, Kiev, $132-35$ (1988).

[35] Gurevitch A.V., Pitaevsky L.P., JETP, 93 (3) 871-880 (1987).

[36] Novikov S.P., a) Funk. Anal. Appl., 24 (4) (1990). b) Progr. in Theor. Phys. (to appear in 1991).

[37] Krichever I.M., Preprint of the Forshungsinstitut fur Math., ETH, Zurich (June 1990).

Landau Institute of Theoretical Physics, Academy of Sciences, Moscow, Russia 